\title{
Gestión de los procesos de la Expo Emprendedores de la Facultad de Contaduría y Administración de la Universidad Veracruzana, utilizando un sistema de información Web
}

Galindo Monfil, Alma Rosa

Universidad Veracruzana, México almgalindo@uv.mx

Martínez Herrera, Brenda Marina Universidad Veracruzana, México brmartinez@uv.mx
Olivares Ruiz, Nancy Araceli

Universidad Veracruzana, México nolivares@uv.mx

Ostos Cruz, Cecilia

Universidad Veracruzana, México ceostos@uv.mx
Resumen - En este documento se sintetiza el trabajo realizado para la construcción de un sistema de información web, el cual automatiza los procesos en el registro de participantes, almacenamiento de información y obtención de resultados de los ganadores de la Expo Emprendedores, de la Facultad de Contaduría y Administración Región Xalapa de la Universidad Veracruzana.

Palabras claves: Sistema de información; web; automatización de procesos; emprendimiento;

Abstract - This document summarizes the work done for the construction of a web information system, applying the Áncora methodology, which automates the processes in the registration, storage of information and obtaining the winners of the Emprendedores Expo, of the School of Accounting and Xalapa Region Administration of the Universidad Veracruzana.
Keywords: Information system; web; process automation; entrepreneurship;

\section{INTRODUCCIÓN}

La Universidad Veracruzana (UV) a través de la Facultad de Contaduría y Administración (FCA), realiza cada año la Expo Emprendedores FCA. Dentro de este evento estudiantes de las licenciaturas de Contaduría, Administración, Gestión y Dirección de Negocios y Sistemas Computacionales Administrativos, que cursan las experiencias educativas desarrollo de emprendedores y diseño de plan de negocios, presentan ideas innovadoras de proyectos en las categorías Tradicional, Tecnología Intermedia,
Interconectando Saberes, 2019

ISSN: 2448-8704
Fecha de Recepción: 20 de septiembre de 2019

Fecha de Aceptación: 24 de octubre de 2019

Fecha de Publicación: 31 de octubre de 2019 
Emprendimiento Social y Alta Tecnología. El presente trabajo consiste en desarrollar un sistema de información web que coadyuve en el registro y evaluación de los proyectos presentados.

Cabe mencionar que se presentan resultados de una investigación aplicada, la cual es entendida como la utilización de los conocimientos en la práctica. De acuerdo con Vargas (2009), la historia de la investigación científica muestra el aprovechamiento de productos teóricos para el diseño de sistemas de acción eficientes para resolver alguna necesidad o situación social deficiente y mejorable de algún modo.

La información como un recurso de las organizaciones Actualmente la información es un recurso trascendental en la vida cotidiana de la sociedad en todos sus ámbitos; es el sustento para la Ilamada sociedad del conocimiento.

Peter Druker introduce el concepto de la "organización basada en la información", sustentado en su es- tudio sobre la evolución de las organizaciones empresariales. Druker observó que las organizaciones aumentan sus niveles gerenciales $y$ sus actividades cada vez más se van fraccionando para lograr la especialización del trabajo (como se cita en Clubensayos, 2013).

Por lo anterior, es claro observar que las organizaciones basadas en la información deben estar en comunicación con el negocio, atender las necesidades de los clientes, los requerimientos del producto, así como los procesos de negocios.

En este sentido, los sistemas de información tienen una gran importancia dentro de las organizaciones basadas en la información, ya que facilitan el flujo de ésta, aceleran los procesos y la comunicación de resultados, asimismo sirven como herramientas para lograr fortalecer la estructura competitiva del negocio, la creación de nuevos productos y servicios, así como la captación efectiva de nuevos clientes y mercados. 
Los sistemas de información como facilitadores de procesos en las organizaciones

De acuerdo con Laudon \& Laudon (2016), "Los cambios en la tecnología, junto con los nuevos modelos de negocios innovadores, transformaron la vida social y las prácticas de negocios". Los Sistemas de Información (SI) son reconocidos como herramientas que facilitan la producción, distribución y uso de la información en las organizaciones, lo cual ayuda a tomar mejores decisiones, generando gestión del conocimiento y

aprendizaje organizacional.

Estos sistemas de información son esenciales en la actualidad ya que permiten lograr los objetivos de negocios estratégicos planteados por los autores Laudon \& Laudon (2016): excelencia operacional; nuevos productos, servicios y modelos de negocios; intimidad con el cliente y con el proveedor; toma de decisiones mejorada; ventaja competitiva, y sobrevivencia.

Por otro lado, aunque una de las principales mejoras que ofrecen los sistemas de información es la auto- matización de muchos pasos en los procesos, en la actualidad con la tecnología de información se ha podido realizar mucho más. Utilizando esta tecnología nueva se puede incluso cambiar el flujo de información de tal forma que varias personas puedan tener acceso a ella y compartirla, remplazando pasos secuenciales con tareas que se puedan llevar a cabo de forma simultánea, con lo cual se eliminan los retrasos en la toma de decisiones.

En este sentido, las Universidades no pueden quedarse atrás $y$ deben incorporar el uso de las tecnologías de información en sus procesos administrativos, así como en la prestación de servicios a la comunidad universitaria.

\section{La Expo Emprendedores FCA}

La "Expo Emprendedores FCA" es un evento que se realiza con la finalidad de promover el espíritu empresarial y de competencia en los estudiantes, teniendo como objetivo principal mostrar los proyectos desarrollados durante el periodo que cursan la experiencia educativa "Desarrollo de Emprendedores". 
Los mejores proyectos de las Licenciaturas en Administración; Contaduría; Sistemas Computacionales Administrativos; y, Gestión y Dirección de Negocios son sujetos a evaluación por parte de empresarios, académicos y autoridades invitados.

La evaluación de proyectos se realiza en dos partes: 1) la presentación escrita de un plan de negocios y 2) la exhibición de un producto o servicio en un stand, así como la evaluación del prototipo.

De acuerdo con la ConvocatoriaFCA (2018), se participa en 4 categorías de negocios:

1) Tradicional. Son aquellos que satisfacen las necesidades básicas de consumo de bienes o servicios de la población en general y se caracterizan porque inician con una inversión pequeña, la oferta se centra en un mercado local y son fáciles de replicar.

2) Tecnología intermedia. Son aquellos que incorporan elementos innovadores considerando las tendencias tecnológicas, se caracterizan por contar con procesos de operación semiespecializados o por emplear tecnología que no se encuentra desarrollada en su totalidad.

\section{3) Emprendimiento sustenta-} ble. Está dirigida al desarrollo de proyectos que satisface las necesidades de cierto sector vulnerable de la población o produce el mayor beneficio posible en la sociedad; tiene un impacto positivo en el desarrollo económico de la región; y realiza una administración eficiente y racional de los recursos naturales sin comprometer la calidad de vida de generaciones futuras.

4) Alta tecnología o base tecnológica. Es una empresa con la capacidad para mantener un flujo de nuevos productos o servicios que satisfacen las demandas del mercado. Tiene un sólido eje de innovación y hacen uso extensivo del conocimiento científico y tecnológico en sectores especializados, tales como tecnologías de la información y comunicación, microelectrónica, sistemas micro electromecánicos (MEM'S), biotecnología, farmacéuticos y nanotecnología. 
Áreas de oportunidad detectadas los procesos de registro y obtención de los ganadores de la Expo Emprendedores

El proceso para la realización de la Expo Emprendedores FCA, se apoyaba en el llenado de hojas de registro manuales, tablas y formatos de Excel, lo que era tardado y muy laborioso, debido a que las inscripciones pueden ser superiores a 30 proyectos. De la misma forma a la hora de emitir los resultados, las evaluaciones tanto internas como externas eran capturadas por 4 personas y 2 escrutadores en el mismo momento del desarrollo del evento, lo que ocasionaba retrasos en la determinación de los ganadores.

\section{Metodología DE LA INVESTIGACIÓN}

La presente investigación de acuerdo a su finalidad es de tipo aplicada, ya que se emplean conocimientos adquiridos en temas de sistemas de información para resolver la problemática de mejorar en el proceso de registro y evaluación de la Expo emprendedores de la Facultad de Contaduría y Administración Zona Xalapa.
Las fuentes de información empleadas fueron documentales y de campo, ya que se consultaron fuentes relacionadas con la expo emprendedor, así como los conceptos y teorías de la disciplina de la ingeniería de software que permitieran determinar una solución al problema planteado. De campo, entrevistando a las personas encargadas de la realización del evento, así como expertos en el área de desarrollo de software.

\section{Metodologías de desarrollo para el sistema web de la Expo Emprendedores \\ Áncora}

Para la fase del análisis de requerimientos del sistema se utilizaron algunas de las herramientas propuestas en la metodología Áncora, la cual cubre la primera etapa para el desarrollo de un nuevo sistema de software y se representa como un guion en una obra de teatro. La forma en la que trabaja Áncora resulta de gran utilidad, ya que fomenta una mayor comunicación con el usuario y que este se involucre en el nuevo software. 
Esta metodología transmite la idea de que, para empezar a trabajar con la elaboración de un nuevo software, se deben tener bases sólidas, a saber: definir correctamente qué se quiere de un software nuevo y tener una representación clara para el usuario y que, además, permita pasar a las siguientes etapas de desarrollo de software de forma sencilla y directa (Sumano, 2001).

\section{Iterativo-Incremental}

En cuanto a la fase de desarrollo (codificación) del sistema se decidió utilizar una metodología ágil: el modelo iterativo incremental, debido al poco tiempo que se tenía para entregar el proyecto. Este modelo de acuerdo con Pressman (2010), permite dar rápidamente cierta funcionalidad limitada de software a los usuarios, a la vez que ésta se puede aumentar en las entregas posteriores del mismo. "El modelo incremental aplica secuencias lineales en forma escalonada a medida que avanza el calendario de actividades. Cada secuencia lineal produce incrementos de software susceptibles de entegarse de manera parecida a los incrementos pro- ducidos en un flujo de proceso evolutivo" (Pressman, 2010). De esta manera, para el sistema de emprendedores se desarrolló el primer incremento el cual fue el producto fundamental, posteriormente se agregaron las demás funcionalidades (incrementos). Otra particularidad de este modelo es la interacción constante con el cliente, su participación y disponibilidad en todo momento. Para el caso del sistema de la expo emprendedores se contó siempre con la Coordinadora del evento, lo que facilitó mucho llevar a cabo este modelo y el desarrollo del proyecto.

\section{Fase de Análisis \\ Construcción de un primer Glosario}

Para realizarlo se buscó información en carpetas, documentos, hojas de Excel, archivos y páginas de internet de los años 2015, 2016 y 2017, con el fin de lograr un mayor entendimiento del contexto y flujo de los procesos de la Expo Emprendedores. En la Tabla 1 se muestra una fracción del glosario elaborado. 


\section{Tabla 1. Fracción de glosario de Áncora}

\begin{tabular}{|c|c|c|c|}
\hline Palabra & Significado & Formas en la que aparece & Referencia \\
\hline Base de datos & $\begin{array}{l}\text { Es una colección de datos } \\
\text { organizada para acceder } \\
\text { fácilmente a la información. }\end{array}$ & Diagrama en forma de árbol. & Hojas de Excel del SUME. \\
\hline Stand & $\begin{array}{l}\text { Es un espacio dentro de una feria, } \\
\text { salón o exposición, donde } \\
\text { presentan productos o servicios. }\end{array}$ & $\begin{array}{l}\text { Carpeta de tamaño pequeño de } \\
3 \mathrm{~m} \times 3 \mathrm{~m}\end{array}$ & $\begin{array}{l}\text { Carpeta Expo-Emprendedor } \\
2015 .\end{array}$ \\
\hline Prototipo & $\begin{array}{l}\text { Es una representación limitada de } \\
\text { un producto. }\end{array}$ & $\begin{array}{l}\text { Puede aparecer desde un trozo } \\
\text { de papel con sencillos dibujos } \\
\text { hasta un complejo software. }\end{array}$ & Página de Internet. \\
\hline $\begin{array}{l}\text { Esquema } \\
\text { conceptual }\end{array}$ & $\begin{array}{l}\text { Es una herramienta de } \\
\text { aprendizaje que se basa en la } \\
\text { representación gráfica de un } \\
\text { tópico a través de la } \\
\text { esquematización de conceptos. }\end{array}$ & $\begin{array}{l}\text { Aparecen de forma jerárquica } \\
\text { dentro de figuras geométricas, } \\
\text { cómo óvalos o recuadros, que se } \\
\text { conectan entre sí, a través de } \\
\text { líneas. }\end{array}$ & Página de Internet. \\
\hline Factor & $\begin{array}{l}\text { Un factor es lo que contribuye a } \\
\text { que se obtengan determinados } \\
\text { resultados. }\end{array}$ & Resultados de una evaluación. & $\begin{array}{l}\text { Documento de la Convocatoria } \\
\text { Expo-Emprendedores FCA } 2015 .\end{array}$ \\
\hline
\end{tabular}

Fuente: Elaboración Propia

\section{Guiones de Áncora}

Áncora utiliza herramientas con adaptaciones en otras áreas distintas de la informática, una de las primeras herramientas que utiliza Áncora es el guion, ya que es un elemento central para esta metodología, con los guiones se podrán obtener los requerimientos de una forma más sistemática y precisa.

\section{Guion de la Situación Actual}

Se obtuvo observando, cómo se trabajaba en el lugar. Se analizó cómo estaba organizado todo el flujo de procesos, sin interrumpir ni opinar en la forma de trabajar de las per- sonas. Se hicieron las anotaciones correspondientes, para así poder determinar las actividades y hasta dónde era factible la automatización de los procesos para el sistema de la Expo Emprendedores. En la Tabla 2 se muestra el guion de la situación actual.

\section{Guion de la propuesta computacional}

Se construyeron cuatro guiones de la propuesta computacional. Cada guion está enfocado a los diferentes roles de usuario del nuevo sistema. En la Tabla 3 se muestra el guion del capturista. 


\section{Tabla 2. Guion de la situación Actual}

Guion: Expo Emprendedores

Escena 1: Entrega de documentos

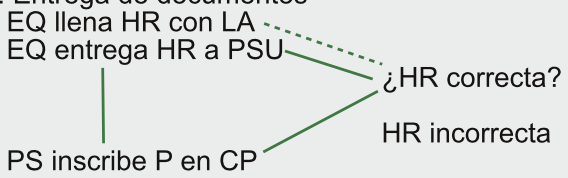

Pista: Control de procesos y obtención Escena 2: Entrega de planes de negocio

del ganador

EQ entrega PN a PSU

PSU guarda PN en SO

Papeles:

$E Q=$ Equipo concursante

EEX = Evaluador externo

EIN = Evaluador interno

PSU $=$ Personal SUME

Útiles:

$\mathrm{P}=$ Proyecto

$\mathrm{PN}=$ Plan de negocios

COG = Constancia de ganadores

$\mathrm{HR}=$ Hoja de registro

$\mathrm{SO}=$ Sobre

$\mathrm{EXC}=$ Excel

LA = Lapicero

FEIN = Formato de evaluación interna

FEEX =Formato de evaluación externa

EXP = Expor Emprendedor

$\mathrm{PRO}=$ Prototipo

STD $=$ Sand

ES = Evaluación del Stand

EP = Evaluación del prototipo

$\mathrm{CP}=$ Categoría del proyecto
Escena 3: Evaluación de planes de negocio

PSU entrega a EIN tres PN

EIN evalúa $P N$ en FEIN

EIN entrega a PS tres PN y tres FEIN

Escena 4: Evaluación de prototipo y stand PSU invita a EEX a participar en EXP PSU entrega FEEX a EEX EEX evalúa PRO y STD en FEEX EEX entrega FEEX a PSU
Condiciones de entrada:

EQ presenta P y $P$ es evaluado

Condiciones de Salida:

$E Q$ ganador recibe COG de EXP

Fuente: Elaboración Propia
Escena 5: Captura de evaluaciones

PSU captura FEIN en EXC

PSU captura FEEX en EXC

Escena 6: Determinación de ganadores

PSU ordena EXC por CP y puntaje de mayor a menor

PSU determina ganadores de EXP con EXC

PSU entrega COG a EQ
Fase de Codificación

Para la codificación del sistema se utilizaron varias herramientas, una de ellas fue el framework de Laravel, el cual fue seleccionado porque permite trabajar con el modelo vista controlador (MVC), lo cual facilita el desarrollo de los sistemas al contar con un código más ordenado y limpio, así como trabajar por separado la interfaz del modelo de datos, reduciendo esfuerzo y tiempo en el equipo del proyecto. Otra herramienta utilizada fue el framework de Boostrap, el cual facilitó el desarrollo de la interfaz y la creación de los CRUD's del sistema web, asimismo se incorporó código de javascript y jquery. Como manejador de Base de datos se utilizó MySQL. 


\section{Tabla 3. Guion del capturista}

Guion: Sistema Expo Emprendedor

Pista: Control de procesos y obtención del ganador

Papeles:

CAP = Capturista

Utensilios:

SEXE = Sistema Expo Emprendedor

$\mathrm{CRE}=$ Clave de registro

$\mathrm{PRO}=$ Proyectos

$\mathrm{CPU}=$ Computadora

$\mathrm{EVI}=$ Evaluadores internos

$\mathrm{EEX}=$ Evaluadores externos

LOG $=$ Login

PROE = Programa educativo

$\mathrm{CP}=$ Categoría proyecto

$\mathrm{EXE}=$ Experiencia educativa

COMP = Componente de evaluación

$E S C=$ Escala de evaluación

$\mathrm{CAL}=$ Calificaciones

$\mathrm{EQ}=$ Equipo concursante

Condiciones de entrada:

CAP llena catálogos de SEXE y asigna

PRO a EVI y EXE

Condiciones de Salida:

Usuarios de EVI y EXE registrados en

SEXE

\section{Fuente: Elaboración Propia}

\section{Fase de Pruebas}

Para esta fase se realizaron los siguientes tipos de pruebas:

- De Unidad: se realizaron pruebas al código de cada módulo para verificar que no existieran errores de compilación y realizaran la función correspondiente.

- De Caja negra: Se probó el funcionamiento de cada módulo verificando sus entradas, el correcto funcionamiento de los procesos y las salidas esperadas. Cabe mencionar que una de las salidas que fueron los reportes, teniendo de forma local el sistema se visualizaron correctamente, sin embargo, ya estando en un servidor los reportes no se pudieron visualizar adecuadamente.

- Del Sistema: se aplicaron pruebas de seguridad, en las cuales se verificó que solo los usuarios dados de alta y con su contraseña correcta pudieran tener acceso, además, que cada usuario solo pudiera ver la vista que le correspondía. Otra prueba que se llevó a cabo fue la de rendimiento, en la cual se revisó que el sistema funcionara correctamente de determinados lapsos de tiempo y ejecutándose varias transacciones a la vez. También se llevaron a cabo pruebas de usabilidad, en la cual algunos alumnos y profesores utilizaron el sistema para verificar su fácil manejo y si la interfaz era 
cómoda e intuitiva, a la vez de evaluar la experiencia de ellos son el sistema. Las pruebas de integración se aplicaron para observar que al unir los módulos éstos no generaran errores y produjeran las salidas correspondientes.

Por otro parte, se realizaron pruebas alfa y beta. La primera consistió en crear un ambiente simulando el evento de le Expo-emprendedores y con un equipo de prueba integrado por alumnos y profesores para utilizar el sistema, el cual para esta etapa se instaló de forma local. En la segunda, el sistema se instaló en un servidor proporcionado por la Dirección de Desarrollo Informático de Apoyo Académico y estuvo en operación del 20 al 30 de noviembre de 2018, para realizar pruebas ya en el ambiente real y sin intervención del equipo desarrollador.

\section{Fase de Implantación}

La primera versión del sistema se instaló con el apoyo de la Dirección de Desarrollo Informático de Apoyo Académico y estuvo en operación del 20 al 30 de noviembre de 2018 , en el servidor proporcionado, aplicando pruebas de accesibilidad y usabilidad principalmente.
- Documentación: Se redactaron los manuales del usuario, así como también los manuales del administrador, capturista, evaluador interno y evaluador externo, estos fueron elaborados para cada uno de ellos. Cada manual fue hecho específicamente para cada rol ya que cada uno cumple diferente función dentro del sistema.

\section{- Método de Conversión: El} método de conversión elegido para la implantación del sistema fue en paralelo para dar mayor seguridad al evento. Se realizó una doble captura en el sistema y en la hoja de cálculo en Excel que se había operado en las versiones anteriores de la Expo Emprendedores, para poder contrastar resultados. Al operar el sistema se constató que cumplía con los requerimientos solicitados y que los resultados emitidos por el sistema fueron los correctos. 
Gestión de los procesos de la Expo Emprendedores...

\section{Módulo de Administración} del Sistema: en él se registran los usuarios y a cada uno se le asigna un rol específico para que tenga ciertos privilegios a la hora de ingresar al sistema, los roles son: administrador, capturista, evaluador interno o evaluador externo (ver Figura 1 y 2).
- El administrador tiene el derecho de acceder a todos los módulos del sistema.

- El capturista tiene el derecho de acceder a la mayoría de los módulos del sistema, con excepción de las evaluaciones del plan de negocios, evaluación de prototipo y evaluación del stand.

Figura 1. Pantalla de inicio del sistema

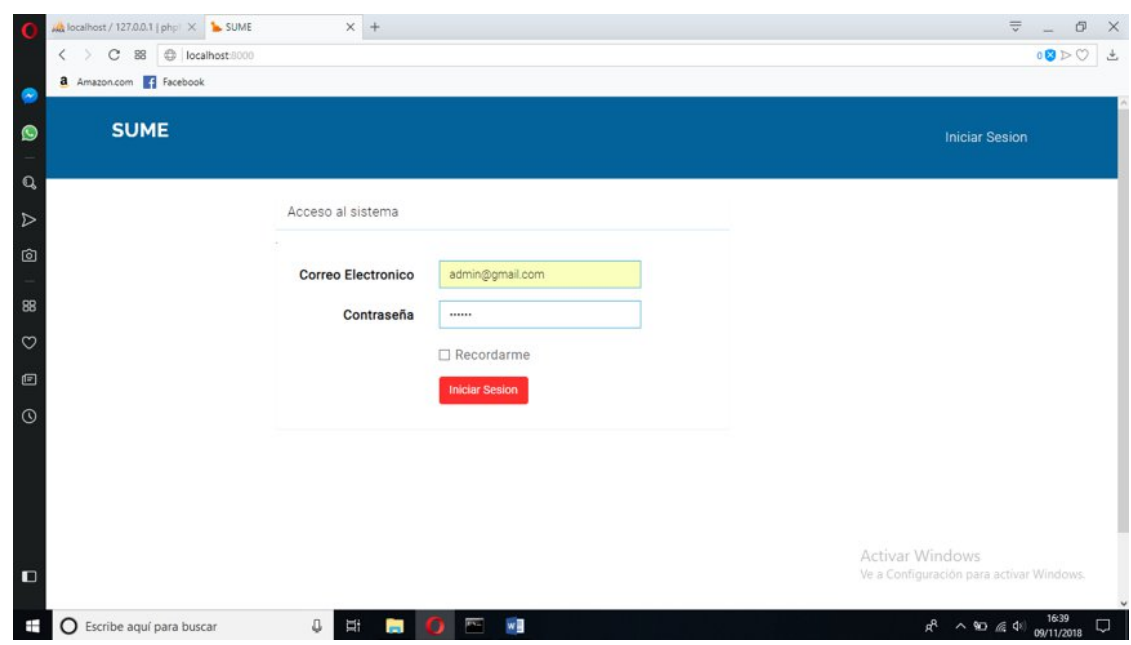

Fuente: Elaboración Propia

Figura 2. Pantalla del menú del administrador

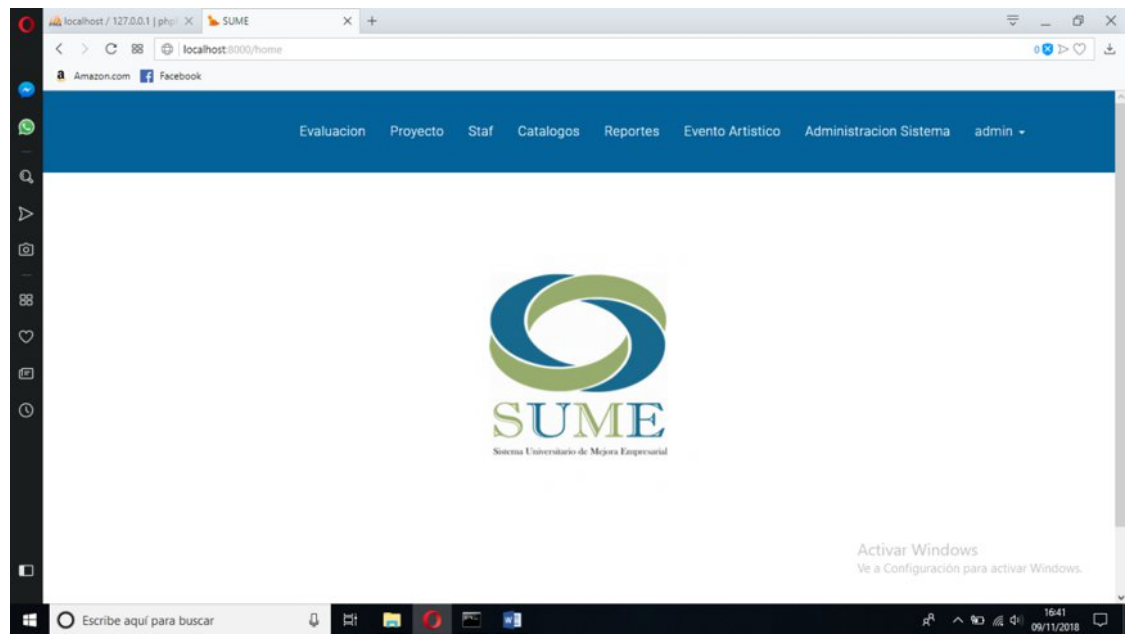

Fuente: Elaboración Propia 
- El evaluador interno solo tiene derecho a acceder al módulo de evaluación del plan de negocios.

- El evaluador externo solo tiene derecho a acceder al módulo de evaluación de prototipo y evaluación del stand.

2. Módulo de Catálogos: permite el registro de la feria, los programas educativos, las experiencias educativas, las categorías de los proyectos, los maestros evaluadores (que son los evaluadores internos), los evaluadores externos, las autoridades invitadas, los componentes del plan de negocio, las escalas del plan de negocios, los criterios del prototipo, las escalas del prototipo, los factores del stand y por último la calificación del stand (ver Figura 3).
3. Módulo de Staff: aquí se realiza el registro de los estudiantes y maestros participantes. De los estudiantes se registran sus datos personales y se les asigna un tipo de participación en la feria, ya sea como organizador o edecán; de la misma forma, se registran los datos personales de los maestros y se les asigna un tipo de participación, ya sea responsable del proyecto u organizador; es muy relevante esta parte, ya que es de suma importancia registrar a quienes van a ser responsables de los proyectos, para poderlos asignar en la inscripción del proyecto (ver Figura 4).

4. Módulo de Proyecto: aquí registra la inscripción de todos los proyectos, consecutivamente se debe realizar la inscripción de los estudiantes

Figura 3. Pantalla del menú catálogo

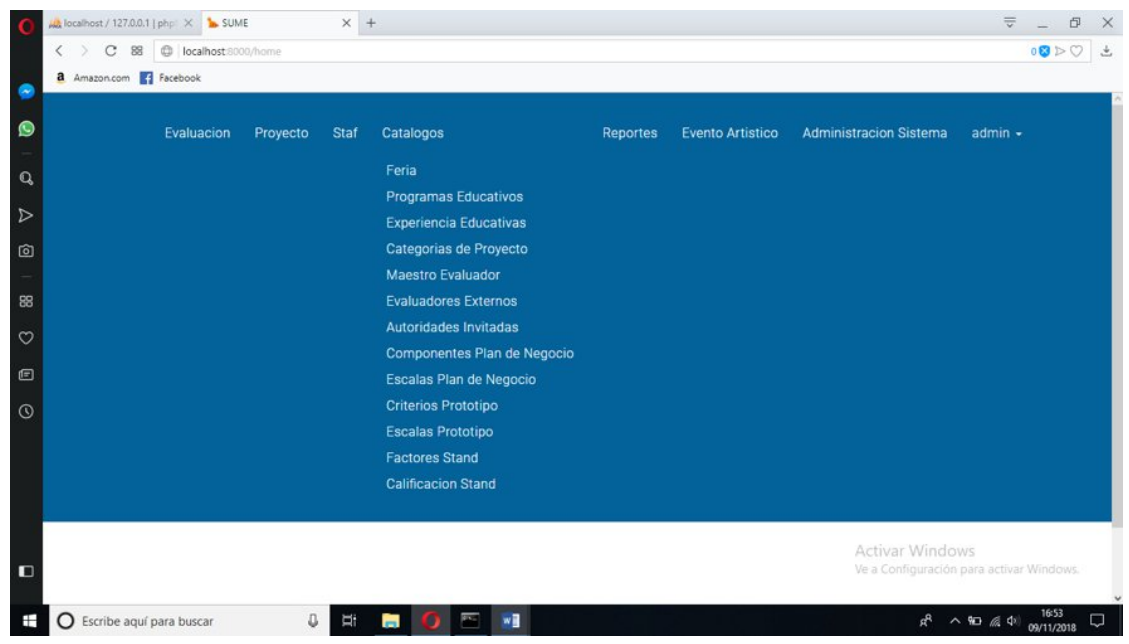

Fuente: Elaboración Propia 
Figura 4. Pantalla del menú Staff

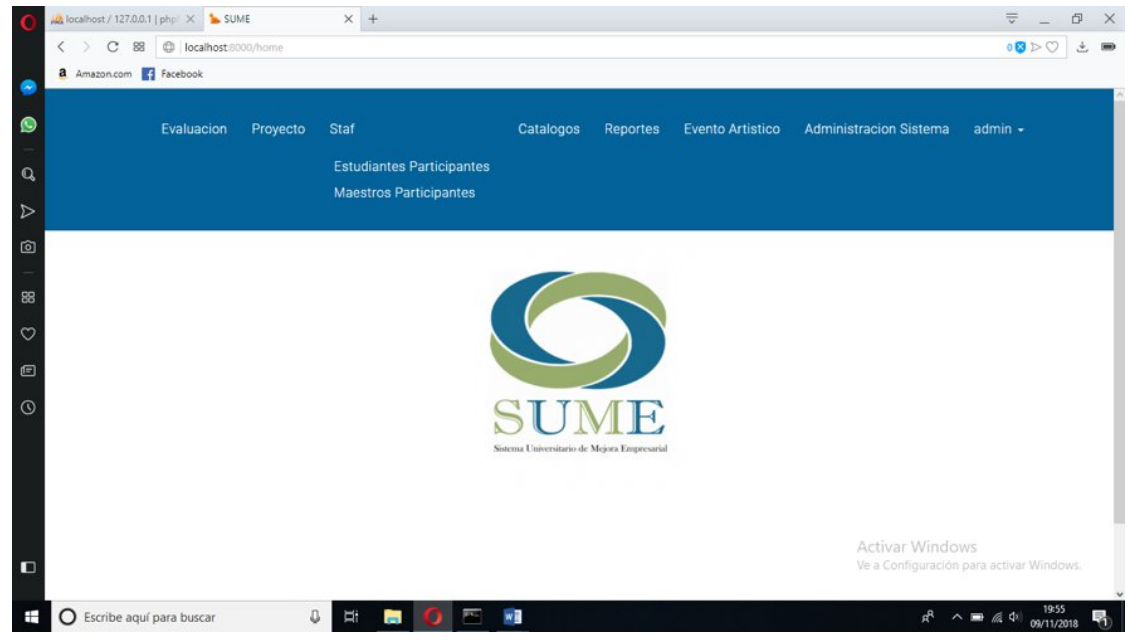

Fuente: Elaboración Propia

concursantes ingresando sus datos respectivos. En la parte superior derecha de cada vista se tiene un apartado de reportes, donde se pueden consultar todos los proyectos y estudiantes concursantes inscritos (ver Figura 5).

5. Módulo de Evaluación: es donde se asigna el evaluador interno, aquí se asignan tres proyectos a cada maestro eva- luador. También se realiza la asignación de evaluador externo y se adjudican los respectivos proyectos a los evaluadores externos; por último, también se tiene acceso a las vistas de: evaluación del plan de negocios, evaluación del prototipo y evaluación del stand, en caso de que el usuario logueado sea el administrador.

Figura 5. Pantalla de menú Proyecto

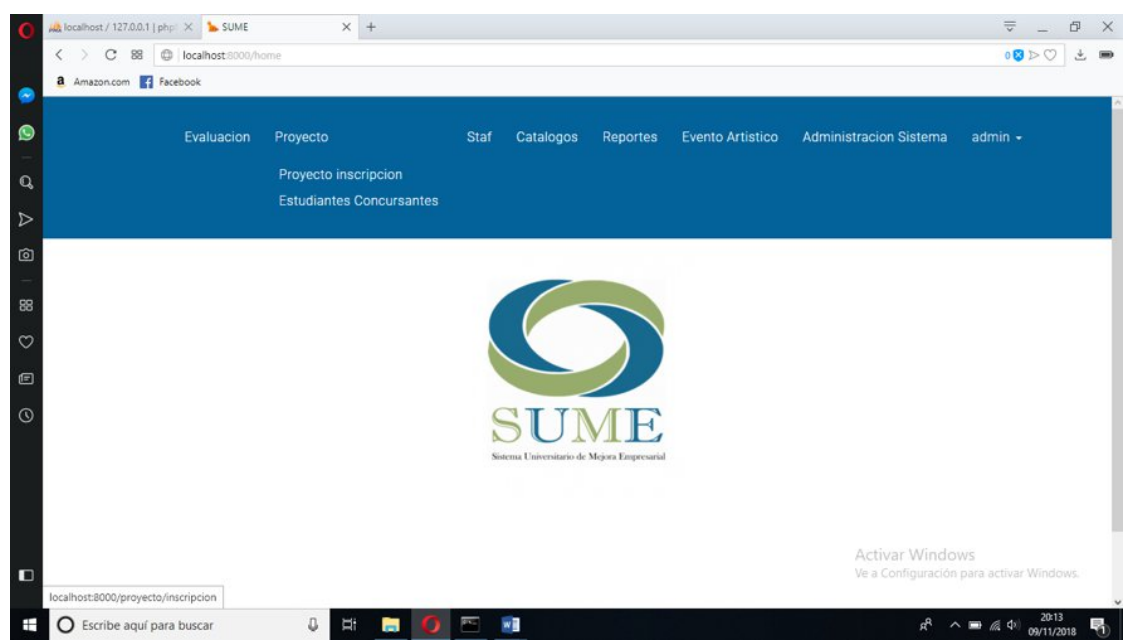

\section{Fuente: Elaboración Propia}


6. Módulo de Evento Artístico: se hace el registro de la información de los grupos artísticos que participan amenizando la Expo Emprendedores.

7. Módulo de Reportes: en los tres primeros reportes llamados plan de negocio, prototipo y stand, se puede consultar la puntuación que obtuvo cada proyecto por categoría y saber qué persona los evaluó. En los siguientes cuatro reportes Ilamados: reporte final, plan de negocios, reporte final de prototipo y reporte final de stand, se puede consultar la puntuación final que obtuvieron y así poder conocer cuáles fueron los proyectos ganadores por categorías de la Expo-emprendedores (ver Figuras 6, 7 y 8).

Figura 6. Reporte de Evaluación plan de negocios

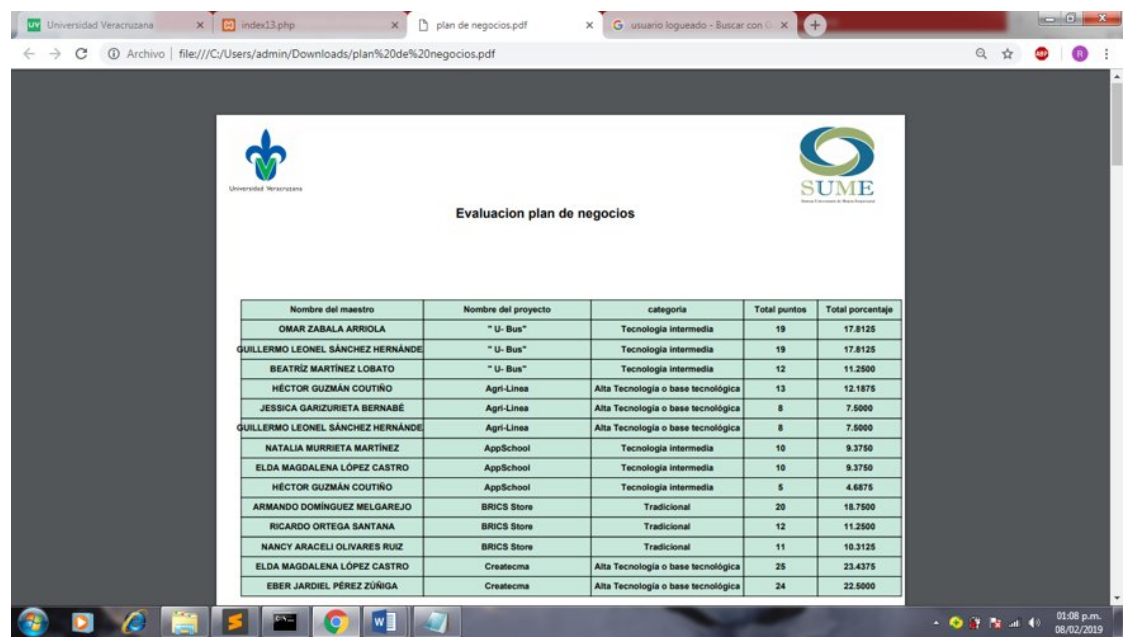

Fuente: Elaboración Propia

Figura 7. Reporte Evaluación de prototipo

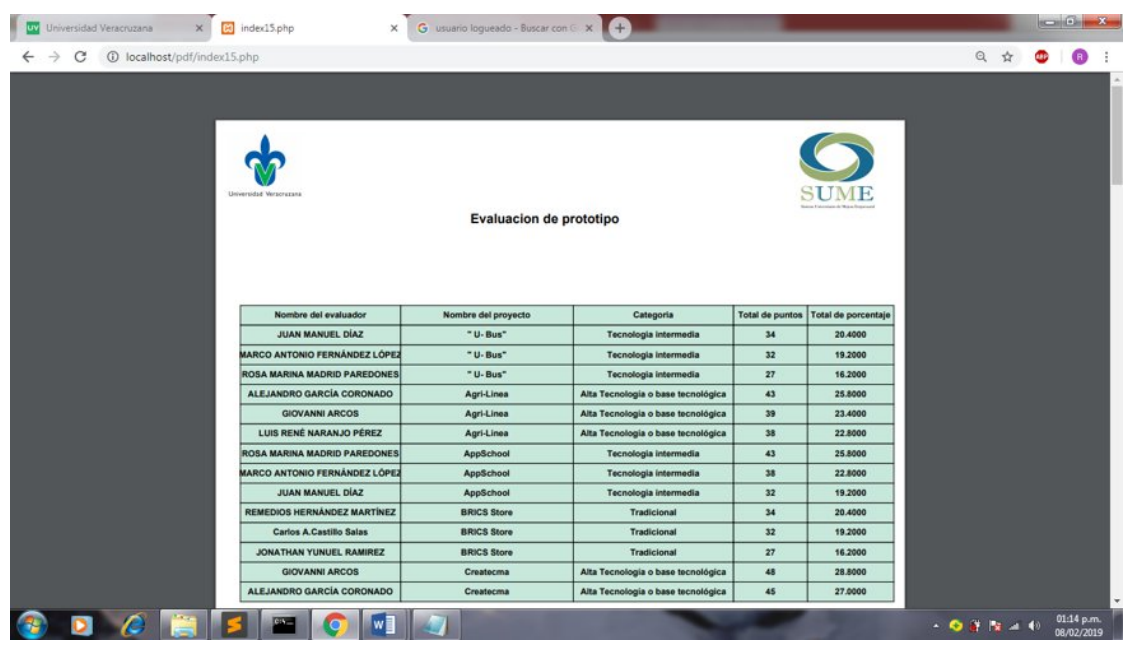

Fuente: Elaboración Propia 


\section{Figura 8. Reporte Evaluación de Stand}

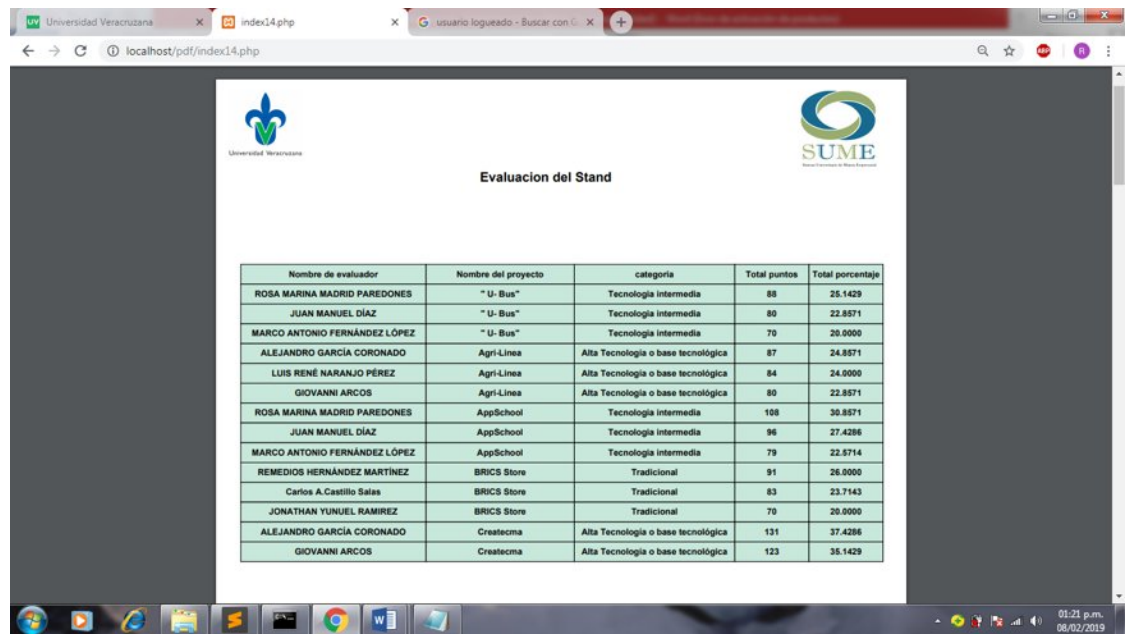

Fuente: Elaboración Propia

Cabe mencionar que la primera versión del sistema fue evaluada por personal de la Dirección de Desarrollo Informático de Apoyo Académico, además se instaló con su apoyo en un servidor que ellos proporcionaron. El sistema estuvo en operación del 20 al 30 de noviembre de 2018, aprovechando los días previos al evento para realizar pruebas del sistema con acceso remoto.

La Expo-emprendedores 2018 se llevó a cabo el día 22 de noviembre de 2018, en el gimnasio de la Unidad de Servicios Bibliotecarios y de Información (USBI).

\section{CONCLUSIONES}

En cuanto al sistema se concluye que cumple con los objetivos del proyecto, ya que apoyó de manera correcta y eficiente en el registro, almacenamiento y obtención de resultados en la evaluación de los proyectos de la Expo Emprendedores de la FCA región Xalapa de la UV.

También se logró que varias personas pudieran tener acceso a la información y poder compartirla de forma simultánea, ya que cada evaluador tanto interno como externo puede ingresar los datos y generar información de forma automática, disminuyendo con esto tiempos en 
la captura por parte de los organizadores y de escrutadores, es decir menos requerimiento de personas en el proceso de captura.

Por otro lado, el hecho de que cada evaluador capturó su información dio mayor confiabilidad en la información generada. Por otra parte, el sistema proporcionó reportes que sirvieron de evidencia para cualquier tipo de aclaraciones. Se obtuvieron resultados de los ganadores del evento de forma mas rápida que en años anteriores.

Por último, cabe mencionar que existen áreas de oportunidad, lo cual implicaría hacer adecuaciones generando una nueva versión del sistema, que permita, por ejemplo, a los evaluadores acceder de manera digital (utilizando dispositivos móviles) a los planes de negocio (utilizando dispositivos móviles) y así evitar la impresión.

\section{REFERENCIAS}

Cardador, C. A. (2014). Implantación de aplicaciones web en entornos internet, intranet y extranet.
Clubensayos. (18 de mayo de 2013). Recuperado el 30 de septiembre de 2019, de

https://www.clubensayos.com/Aconte cimientos-Sociales/La-OrganizacionBasada-En-La-

Informacion/772738.html

Convocatoria-FCA. (2018). Obtenido de

https://www.uv.mx/fca/files/2018/11/C onvocatoria-FCA-2018.pdf

Convocatoria-FCA. (2018).

Convocatoria-FCA. Obtenido de https://www.uv.mx/fca/files/2018/11/C onvocatoria-FCA-2018.pdf

Hermida, C. (2018). UV realizó Expo Emprendedores FCA 2018.

Universo. Sistema de noticias de la UV. Recuperado de https://www.uv.mx/prensa/relevantes/ uv-realizo-expo-emprendedores-fca2018/

Laravel, D. d. (29 de Septiembre de 2013). Introduccion - Documentation Laravel PHP Framework. Obtenido de https://laravel.com/

Laudon , K. C., \& Laudon , J. P. (2016). Sistemas de información gerencial (Decimocuarta ed.). México: PEARSON EDUCACIÓN.

Muñoz, J. F. (s.f.). Ventajas de las aplicaciones web. Obtenido de https://www.pixima.net/aplicacionesweb/ventajas-de-las-aplicacionesweb/

Pressman, R. S. (2010). Ingeniería del software. Un enfoque práctico (Séptima ed.). (P. R. Vázquez, Ed., \& V. C. Olguín, Trad.) México: Mc Graw Hill. 
Sumano, M. d. (2001). Áncora:

Metodología para el Análisis de

Requerimientos de Software

conducente al Reuso. Obtenido de

uv.mx personal:

https://www.uv.mx/personal/asumano

/files/2010/07/Guia.pdf

SUME. (2018). Reseña Expo

Emprendedores FCA. XALAPA.

Universidad Veracruzana FCA. (2019).

Facultad de Contaduría y

Administración. Obtenido de

https://www.uv.mx/fca/sume/sume/

Vargas, Z. (2009). La investigación

aplicada: una forma de conocer las

realidades con evidencia científica.

Revista Educación, 33(1), 155-165.

Recuperado de

https://www.redalyc.org/articulo.oa?i

$d=44015082010$ 